\title{
Aplicación móvil integrada para la supervisión del proceso de producción del banano
}

\section{PIntegrated mobile application for the supervision of the banana production process}

Washington Antonio Caraguay Universidad Espíritu Santo (Ecuador) jcordero@uees.edu.ec

Jorge Cordero Universidad Espíritu Santo (Ecuador) wcaraguay@uees.edu.ec

Revista Cumbres Vol.4 №1

Versión impresa ISSN 1390-9541

Versión electrónica ISSN 1390-3365

http://investigacion.utmachala.edu.ec/revistas/index.php/Cumbres 


\title{
RESUMEN
}

La presente investigación tuvo como objetivo evaluar los componentes nutriEn el presente artículo se ha desarrollado un sistema integrado para supervisar el proceso de producción del banano durante las fases de cosecha y empaque, haciendo uso de tecnologías móviles con base en la información recolectada. La arquitectura del sistema se basa en la obtención de datos desde una estación de pesaje electrónica a través de un dispositivo de entrada móvil. Haciendo uso del Internet, los datos son transmitidos de manera inalámbrica a un servidor local, el mismo que los procesa y envía a un dispositivo final de usuario para su lectura. Los resultados logrados permiten obtener información relevante del proceso que debe ser analizada para la toma de decisiones necesarias para contribuir en el incremento de la productividad en las haciendas bananeras.

Palabras clave: tecnologías móviles, supervisar, proceso de producción del banano, estación de pesaje electrónica.

\begin{abstract}
This work reports the results of the development of an integrated system to supervise, using mobile technologies, the banana production process during the harvesting and packing phases. The architecture of the system is based on obtaining data from an electronic weighing station through a mobile input device. Using the Internet, the data is transmitted wirelessly to a local server, which processes it and sends it to an end user device for reading. The results gathered allow to obtain relevant information of the process. Such information must be analyzed for making the necessary decisions and contribute in the increase of the productivity in the banana plantations.
\end{abstract}

Keywords: mobile technologies, supervise, banana production process, electronic weighing station.

\section{INTRODUCCIÓN}

A inicio de los años noventa el sector agrícola en Latinoamérica se encontró en la necesidad de buscar mecanismos que les permitan ser más competitivos por la influencia de la globalización en los mercados. Existió un incremento significativo en la oferta mundial del banano especialmente desde los países productores de centro américa, según Soto (2011), "la oferta mundial pasó de 5.8 millones de toneladas en el periodo 1983-85 hasta 10.7 millones de toneladas en el período 1993-95, donde el país que más creció fue Guatemala con un $101.7 \%$, seguido por Ecuador con un $12.44 \%$ y Costa Rica con un 7.89\%" (p.14). Uno de los mecanismos que ya se utilizaban en países de otras

\section{2}


zonas del mundo y que se implementó fue el uso de la tecnología. Primero se fundaron empresas de desarrollo de software dedicado a la administración y gestión de empresas agrícolas lo cual ayudó a un mejor manejo de información, luego se desarrollaron softwares para el manejo de información ya no administrativa sino del proceso de producción, esto ayudó a las empresas a encontrar puntos débiles en la cadena de producción y poder mejorarlos llegando a ser más productivos.

Sin embargo, durante los últimos años el Ecuador ha sido menos efectivo que sus competidores directos por algunos factores tanto internos como externos. Uno de los más relevantes es la globalización que regula los precios de los productos en los mercados, nuestros competidores directos desde hace algunos años son Costa Rica, Honduras y Guatemala, países centroamericanos que tienen la ventaja de estar cerca del canal de Panamá, lo cual disminuye los costos de transporte y desde hace pocos años tenemos como competidores a nuestros vecinos Colombia y Perú que tienen la ventaja de tener mano de obra más barata como podemos observar en la Figura 1, "el comparativo de precios de salida FOB (Free On Board) entre Ecuador, Colombia, Honduras y Costa Rica es de 7.25 dólares hasta 2011, el más alto es de Costa Rica y el más bajo es el de Colombia" (Vega Jaramillo, 2015).

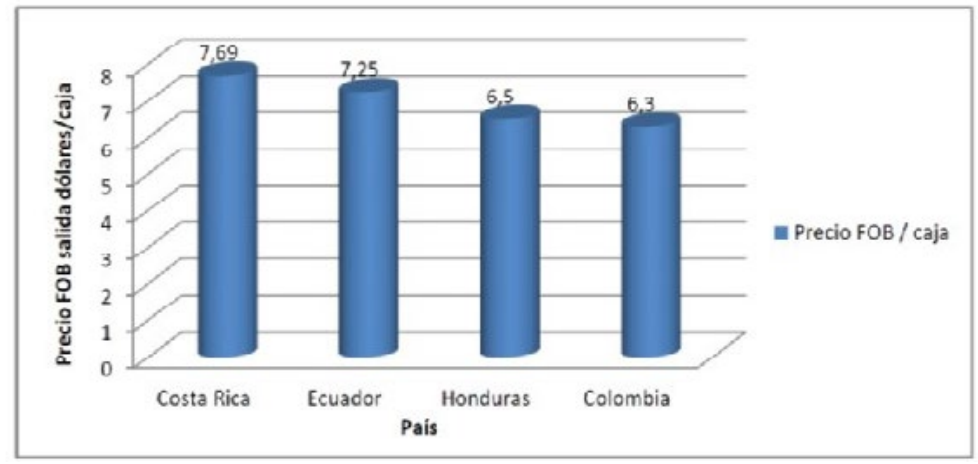

Figura 1: Precio FOB de la caja de banano en el mercado internacional Fuente: (Vega Jaramillo, 2015)

La exigencia de los mercados mundiales de aumentar la calidad de la fruta, ha obligado a realizar cambios en todo el proceso productivo: cosecha, empaquetamiento, transporte interno desde las plantaciones hasta los puertos marítimos y transporte marítimo internacional, hasta llegar a las perchas de los mercados mundiales. Toda esta logística generó un aumento en el costo de producción. Otro factor importante que va de la mano con el aumento de la calidad que exigen los mercados internacionales es la implementación de Certificaciones Internacionales de Exportación. Todos estos factores han llevado a los productores difícilmente a buscar maneras de reducir costos y de ser más productivos.

Como consecuencia de los factores expuestos, los productores han implementado diferentes herramientas para el control de calidad, entre los cuales está el software de control, el mismo que ha ido evolucionando e integrando 
los procesos de producción y administración. Así, con la intervención de las redes de datos, el Internet y las aplicaciones móviles, surgen los ERP (Enterprise Resource Planning), los mismos que según (Logismic Software, 2016), son conjuntos de sistemas informáticos que permiten integrar ciertas áreas de una empresa como administración, producción, logística, entre otros. Además, la presencia de empresas que actualmente se dedican al desarrollo de software como Agrosoft (Agrosoft, 2016), una compañía ecuatoriana con varios años de experiencia, otro es el caso de la compañía colombiana Biosalc (Biosalc, 2016), y también están las grandes compañías mundiales como Bayer (Bayer, 2016), Syngenta y Monsanto que dedican tiempo y recursos para la creación de nuevas herramientas para ayudar al productor agrícola. Existen aplicaciones móviles con información sobre todo tipo de cultivo y su manejo. Existen también otras grandes empresas mundiales dedicadas al desarrollo de tecnologías que usan software de control y supervisión agrícola como las redes de sensores en el caso de la compañía estadounidense Irrometer (Irrometer, 2016), drones en el caso de SenseFly (Elika, 2016), el auto guiado en el caso de John Deere (John Deere, 2016), y existen pequeñas empresas de emprendimiento que se dedican a la incorporación de la robótica aplicada a los distintos procesos agrícolas como la siembra, deshoje, fertilización y cosecha (Priva, 2016). Sin embargo, uno de las mayores limitantes en la adquisición de estos sistemas es su elevado costo y la instalación de módulos adicionales aumenta considerablemente su precio, quedando fuera de las manos de los pequeños productores, que dentro del Ecuador son un número elevado, por ejemplo solo en la provincia del Guayas, hasta agosto del 2012 existían 3150 productores que tenían desde menos de 5 hasta 20 hectáreas en producción, comparado con los 710 productores que tenían más de 20 hectáreas, en este caso los pequeños productores abarcarían el 81\% del total de productores en el Guayas (Agroban, 2012).

Tomando en cuenta el impacto positivo que tiene la utilización de tecnologías en los procesos productivos, el impacto de la inversión en tecnología sobre la productividad, expone que por cada dólar invertido en Tecnologías de la Información, se producen incrementos de entre 0.81 y 2.62 dólares en el producto de la empresa (Lopez Sanchez, 2004), tomando en cuenta la afirmación expresa del Abg. Eduardo Ledesma, Director Ejecutivo de AEBE (Asociación de Exportadores de banano del Ecuador), indica que "la utilización de tecnologías es esencial para mejorar la eficiencia productiva y la competitividad", y tomando en cuenta las afirmaciones del Prof. Moisés Soto Ballesteros, una de las máximas autoridades académicas y científicas en el área bananera de la Universidad Earth de Costa Rica, indica que "la agricultura de precisión como herramienta de toma de decisiones nos permite tomar acciones correctivas a tiempo y mejorar la productividad" (Soto, 2011). Así con la presente investigación, se busca la manera de utilizar los medios disponibles para proporcionar a los productores bananeros un sistema informático basado en software que provean información del proceso de producción del banano que permita tomar decisiones para mejorar la calidad y productividad bananera. 


\section{MATERIALES Y MÉTODOS}

Lo que se investiga es una manera de mejorar el proceso de producción del banano mediante el desarrollo de una aplicación informática que consiste en conectar un dispositivo móvil con una balanza electrónica para obtener un dato digital de su masa y poder guardarlo en una base de datos, conjuntamente con más datos relevantes del proceso productivo. Dicha aplicación está destinada a la toma de datos en la estación de pesaje que se ubica al inicio de la planta empacadora, en este punto los racimos de banano llegan desde el campo y son registrados junto con sus características físicas. La información tomada es procesada y entregada en forma de reporte. Se aborda solamente esta parte del proceso de producción ya que la información tomada en este punto nos muestra los principales índices productivos del campo.

Para la infraestructura física del desarrollo del sistema, es necesario mencionar las siguientes características: Es necesario instalar en el sistema de transporte de la fruta (vía cable) la celda de carga de la balanza electrónica. La balanza a su vez debe estar conectada en un punto eléctrico para su funcionamiento, debido a la permanencia de su operación, al igual que una Tableta móvil. Se debe contar con un Access Point cercano para que la Tableta esté conectada a la red de Internet.

Estructuralmente el diseño de la investigación comienza con una búsqueda exhaustiva de información de los software de control y supervisión agrícola, con base en esta información se pudo determinar que existen pocas empresas tanto locales como nacionales dedicadas al desarrollo de estos servicios. Se determinó también que existe muy poca tecnología aplicada a los procesos de producción agrícola que permitan aumentar la eficiencia de dichos procesos. Con base a la necesidad de automatizar el proceso de toma de datos de los parámetros físicos que se realiza en el punto inicial del proceso de empaque del banano se desarrolla la presente propuesta, que consiste en la implementación de una estación de medición de la masa de racimos de banano que permite tomar el dato de la masa de cada uno de los racimos que ingresan al proceso de empaque mediante un transductor mecánico eléctrico, además del origen de cada uno de ellos tomando como referencia la lotización interna de la plantación, seguido de esto permite registrar las características particulares ocasionadas por plagas y por labores de campo mal realizadas, toda esta información es almacenada en una base de datos que se encuentra en la red interna y que permitirá procesar esta información y presentarla en forma de reportes.

La arquitectura funcional del sistema se basa en la obtención de los datos desde la estación de pesaje a través de la Tableta digital, que de manera inalámbrica envía estos datos al servidor local, el mismo que los procesa y haciendo uso del internet los envía a un dispositivo final de usuario para su lectura. En la Figura 2, se muestra un esquema de la arquitectura del sistema implementado. 


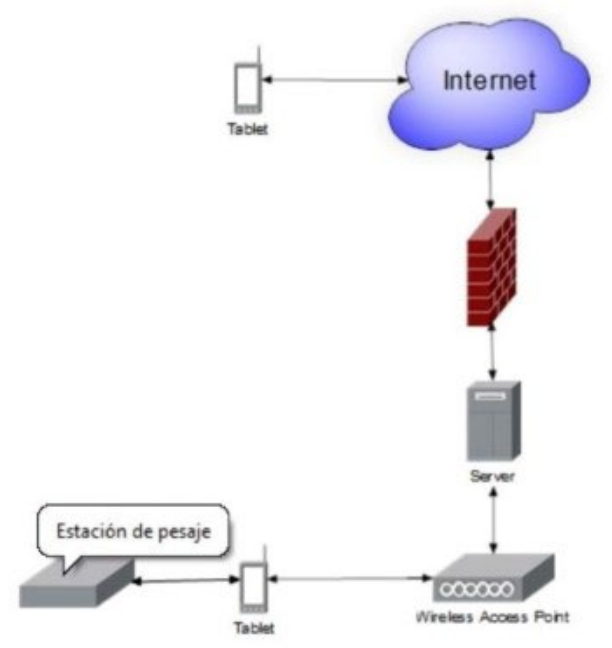

Figura 2: Arquitectura Funcional del sistema integrado de supervisión.

Para la implementación física del sistema, se utilizan diferentes componentes tecnológicos, los cuales son escogidos y diseñados para ser utilizados en los procesos de toma y almacenamiento de la información generada, los mismos que son descritos a continuación.

En la implementación de la estación de pesaje de racimos de banano se utiliza una báscula electrónica Marca Torrey modelo EQB-50/100 (Torrey, 2016). Este módulo, es el encargado de alimentar y recibir la señal de la celda de carga por la interfaz RJ11 y presentar la medida de la masa en el display. Para la conversión de datos entregados por el Módulo EQB en protocolo RS232 a Bluetooth, se diseñó una tarjeta de enlace haciendo uso del integrado MAX232. De esta manera, los datos son recibidos por la Tableta móvil. El diagrama esquemático y la tarjeta ensamblada son mostrados en la figura 3.

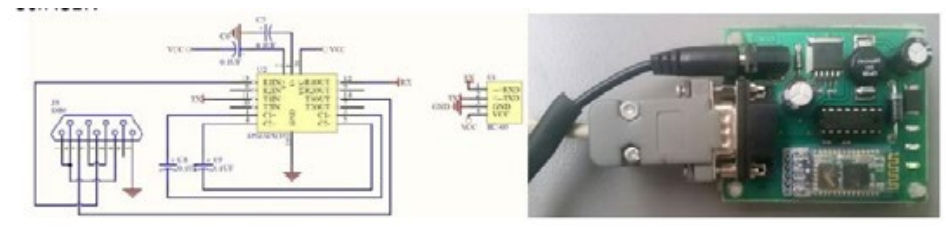

Figura 3: El diagrama esquemático y tarjeta ensamblada del módulo de enlace.

Se realizó un muestreo de las masas de los racimos en tres días del proceso de producción, para establecer la capacidad de la celda de carga a utilizar, la Tabla 1 muestra dicha información. De esta manera, la celda de carga de la balanza a utilizar tiene una capacidad máxima de carga de 100 lbs, una incertidumbre de medición de 0.02 libras y su temperatura de operación óptima está entre $0^{\circ} \mathrm{C}$ y $40^{\circ} \mathrm{C}$. Con base en los requerimientos del sistema se diseña una base de datos utilizando el sistema PostgreSQL. Así, según los requerimientos del sistema se deben realizar dos procesos: el primero donde 
se ingresa información al sistema, y el segundo donde se recibe información desde el sistema.

Tabla 1: Medida de la masa de racimos muestreados en libras, hacienda Catay

\begin{tabular}{lccc}
\hline & \multicolumn{3}{c}{ Días de producción } \\
\hline Racimos muestreados $^{*}$ & 10 -may & 12 -may & 13 -may \\
Masa promedio de racimos (lbs.) & 192 & 153 & 155 \\
Masa Mínima (lbs.) & 70.4 & 68.8 & 67.9 \\
Masa Máxima (lbs.) & 37.4 & 28 & 29.2 \\
\hline
\end{tabular}

Nota: *Indica la cantidad de racimos que fueron pesados por día

La información que se debe ingresar al sistema se detalla a continuación:

1. Lote: Porción de terreno donde se cosechan los racimos.

2. Número de viaje: el número ordinal del grupo de racimos cosechados.

3. Número de racimos: número ordinal de cada racimo en su grupo.

4. Masa del racimo: En libras de cada racimo.

5. Color de cinta: color del indicador de la edad del racimo, según el calendario bananero.

La primera fase del proceso de ingreso de información de racimos a la base de datos se observa en el diagrama secuencial de la Figura 4.

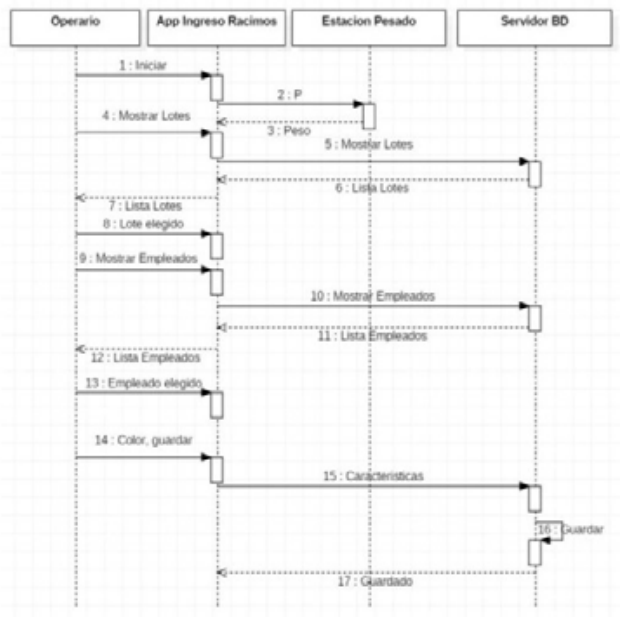

Figura 4: Diagrama secuencial de ingreso de información al Servidor. 


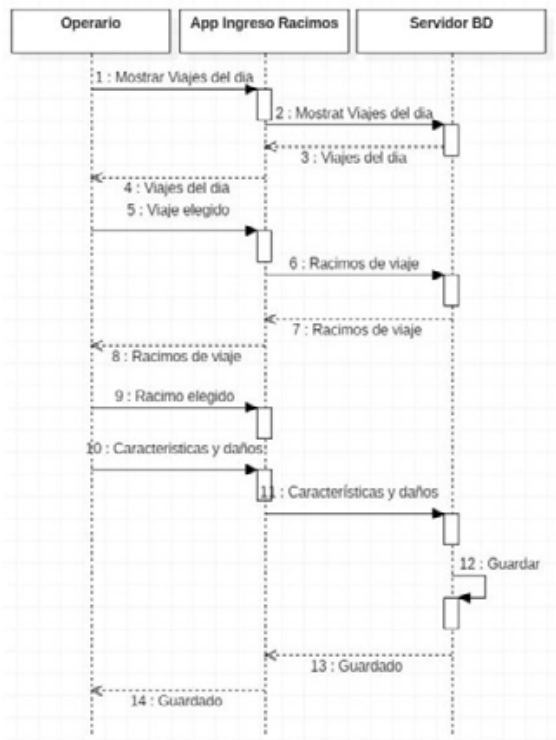

Figura 5: Diagrama secuencial de ingreso de daños por racimo

La fase del proceso de ingreso de información de racimos se observa en el diagrama secuencial de la Figura 5.

Así, La información que se recibe desde el sistema es la siguiente:

- Cantidad de racimos cosechados por lote

- $\quad$ Porcentaje de incidencia de plagas por lote

- Masas promedio de racimos por lote

Para el almacenamiento de información del sistema, se utilizó un servidor por la cantidad de información generada, debido a que la cosecha en haciendas bananeras es constante durante todas las semanas del año, por ende la información se genera en grandes cantidades

\section{RESULTADOS Y DISCUSIÓN}

Pruebas de Funcionamiento

El montaje de la parte física de la balanza del sistema de supervisión se

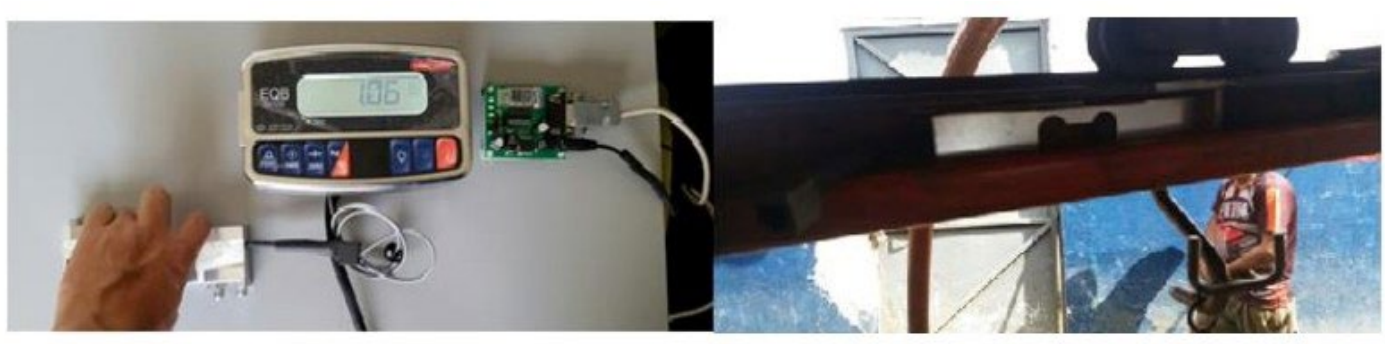

Figura 6: Montaje físico y ubicación de la balanza del sistema de supervisión

\section{Cumbres}


muestra en la figura 6, en la cual se observan: el módulo EQB, la celda de carga y la tarjeta de enlace

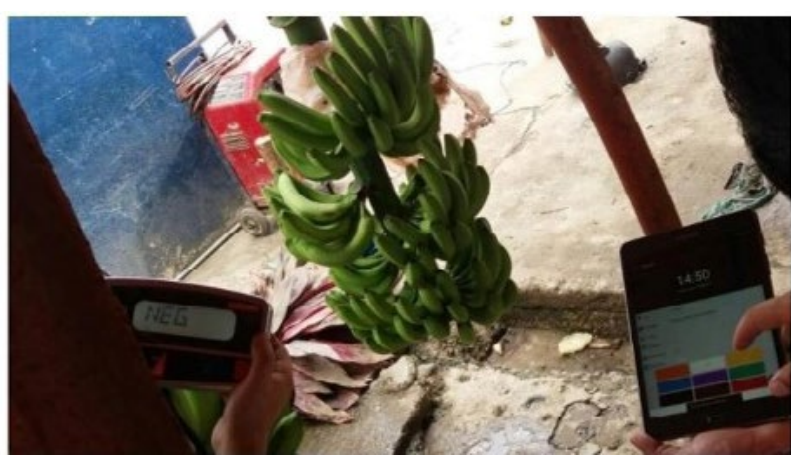

Figura 7: Prueba de funcionalidad del sistema

Para la comprobación del sistema, se utiliza un racimo de banano, se prueba la conectividad entre todos los dispositivos y aplicaciones, los cuales se comunican e intercambian información de manera correcta. La figura 7, muestra una prueba de funcionalidad del sistema.

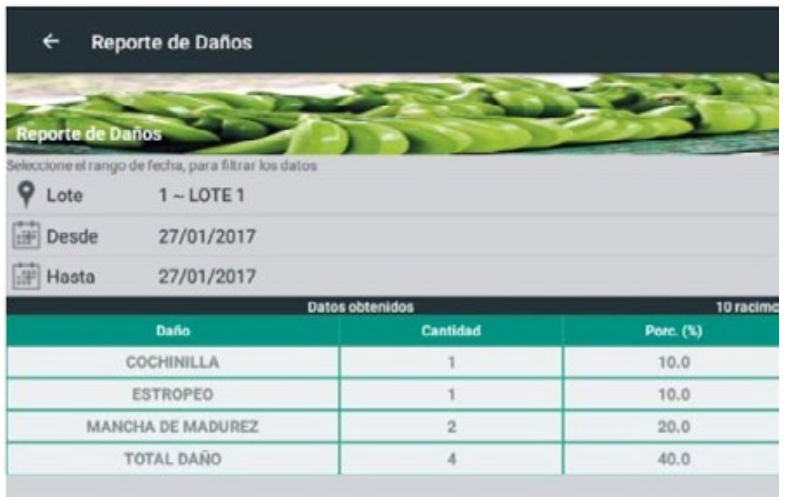

Figura 8: Porcentaje de incidencia de daños en racimos por lote.

Se realiza un ingreso de datos, donde se analizaron 10 racimos de un lote, luego se realiza una consulta al servidor el cual entrega los porcentajes de incidencia de los daños existentes, como lo muestra la figura 8.

Luego de analizar los componentes utilizados para el desarrollo del sistema y su funcionalidad se observa que el costo de la implementación es bajo, ya que permiten procesar información que contribuirá a elevar la productividad de las haciendas bananeras, Tabla 2.

Realizadas las pruebas del mismo se observa que su funcionamiento en parte contribuye con las necesidades de una hacienda bananera en obtener de manera confiable y de fácil acceso, información que es esencial para la mejora del proceso de producción del banano. Además, se almacena la infor- 
mación dentro de una base de datos lo que permite crear un histórico del manejo de la hacienda para futuros análisis necesarios, para que la administración pueda tomar decisiones y acciones correctivas de manera rápida y eficaz con base a datos que reflejan la realidad de la calidad de la productividad de la hacienda.

Tabla 2: Costos de implementación.

\begin{tabular}{|c|c|}
\hline Costos & Valor \\
\hline Tableta Samsung Galaxy Tab A 8" WiFi 16 Gb & $\$ 350.00$ \\
\hline Balanza de recibo Torrey modelo EQB 50/100 & $\$ 280.32$ \\
\hline Tarjeta de enlace RS-232 Bluetoth & $\$ 150.00$ \\
\hline Servidor & $\$ 1,000.00$ \\
\hline Mano de obra* & $\$ 8,000.00$ \\
\hline & $\$ 9,780.32$ \\
\hline
\end{tabular}

Nota: *Suma de costos de desarrollo de software, logística e implementación. Fuente: Elaboración propia.

El costo de implementación del sistema es aproximadamente 10000 dólares, si a este valor se divide para el costo de la caja de banano que es de 6.26 dólares para el año 2017, se obtiene la cantidad de cajas que una finca debería producir adicional a su producción normal para cubrir la inversión, el valor obtenido es de 1597 cajas. En Ecuador el promedio de producción de cajas por hectárea al año en 2016 fue de 1938 (Pro Ecuador, 2016) tomando en cuenta a pequeños, medianos y grandes productores, con base en esta información se obtiene el porcentaje de aumento de producción que debe ser para cubrir la inversión, Tabla 3.

Para que un productor de 5 hectáreas cubra la inversión en 1 año debe aumentar un $22.17 \%$ su producción y un productor de 10 hectáreas debe aumentar un $8.17 \%$ su producción. Para el caso de un productor de 100 hectáreas debe aumentar solo un $0.83 \%$. Con base a lo expuesto y la información del marco teórico, en el Ecuador hay fincas con producciones altas que llegan a las 3500 cajas por hectáreas por año, y que para un productor de 5 hectáreas llegar a esos rendimientos supondría un aumento del $142 \%$, se puede decir que es viable para todos aumentar y sobrepasar el porcentaje necesario para cubrir la inversión del sistema.

\section{CONCLUSIONES}

El sistema desarrollado consiste en una herramienta tecnológica con componentes que se encuentran disponibles en el medio, cuya interfaz de usuario desarrollada es de fácil operatividad, que se acopla al medio en el que se realiza el proceso de producción, requiriendo una ligera capacitación 
en su uso y funcionamiento.

Tabla 3: Rendimiento promedio por hectárea en Ecuador y porcentaje de aumento para cubrir inversión.

\begin{tabular}{cccc}
\hline Hectareaje & Productividad Cajas/Ha/año & Total cajas/año & \% Aumento \\
\hline $\mathbf{5}$ & 1441 & 7205 & $22.17 \%$ \\
$\mathbf{1 0}$ & 1955 & 19550 & $8.17 \%$ \\
$\mathbf{2 0}$ & 1999 & 39980 & $3.99 \%$ \\
$\mathbf{5 0}$ & 1932 & 96600 & $1.65 \%$ \\
$\mathbf{1 0 0}$ & 1932 & 193200 & $0.83 \%$ \\
$\mathbf{2 0 0}$ & 1932 & 386400 & $0.41 \%$ \\
\hline
\end{tabular}

Nota: *Es el porcentaje de aumento en la producción, para cubrir la inversión. Fuente: (Pro Ecuador, 2016)

El sistema de aplicación móvil implementado, representa una contribución más en el desarrollo de tecnologías aplicadas al proceso de supervisión de la producción agrícola, en este caso direccionado al procesos de cosecha y empaque, logrando obtener información que permita tomar decisiones para mejorar la calidad del banano. El análisis financiero indica que el costo de los componentes tecnológicos usados en la implementación del sistema es bajo, ya que son componentes comunes que se utilizan en el medio, esto permite que esté al alcance de muchos productores bananeros que serán beneficiados con el sistema.

Debido a que el sistema es de fácil escalabilidad, se sugiere a futuros investigadores, se instalen tabletas digitales con aplicaciones diferentes en otros puntos del proceso de empaque, por ejemplo una tableta en el área de análisis de merma, otra en el área de repesado junto con otra balanza electrónica para registrar las cajas que son procesadas con sus características, y una última en el área de paletizado, para registrar los contenedores y otros transportes utilizados para la entrega de la producción.

\section{REFERENCIAS BIBLIOGRÁFICAS}

Agroban. (Agosto de 2012). Corporación Regional de bananeros Ecuatorianos. Recuperado el 2 de Diciembre de 2013, de http://agroban.com.ec/ wp-content/uploads/2012/Distribucion_bananera_Guayas.pdf

Agrosoft. (2016). agrosoft.com. Recuperado el noviembre de 2016, de http:// agrosoft.wixsite.com/agrosoft

Bayer. (2016). www.bayer.com. Recuperado el 8 de enero de 2017, de http:// www.bayer.com/en/bayer-apps.aspx

Biosalc. (2016). biosalc.com. Recuperado el mayo de 2016, de http://biosalc. com/soluciones/

Elika. (2016). elika.eus. Recuperado el octubre de 2016, de http://www.elika.eus/datos/articulos/Archivo1388/Berezi\%2035\%20drones\%20y\%20 
sus\%20usos\%20en\%20agricultura.pdf

Irrometer. (2016). irrometer.com. Recuperado el octubre de 2016, de http:// www.irrometer.com/default.htm

John Deere. (2016). deere.com.mx. Recuperado el noviembre de 2016, de https://www.deere.com.mx/es_MX/services_and_support/tips/ams/innovation_on_agriculture/innovation_agriculture.page?

Logismic Software. (2016). sistemaagricola.com.mx. Recuperado el septiembre de 2016, de http://sistemaagricola.com.mx/

Lopez Sanchez, J. I. (2004). Pueden las tecnologías de la información mejorar la productividad? Universia Business Review, 85-86.

Priva. (2016). priva-international.com. Recuperado el noviembre de 2016, de http://www.priva-international.com/en/discover-priva/stories/2016/priva-kompano-deleaf-line/

Pro Ecuador. (2016). Producción Nacional . Análisis Sectorial Banana 2016, 6.

Soto, M. (2011). Situación y avances tecnológicos en la producción bananera mundial. Revista Brasileña de Fruticultura, 14.

Torrey. (2016). www.basculas-torrey.com. Recuperado el 2 de junio de 2016, de http://basculas-torrey.com/descargas/catalogo-refacciones/eqb-Refacciones.pdf

Vega Jaramillo, V. L. (2015). Análisis de los precios de la caja de banano y su impacto en los ingresos de los productores bananeros. Guayaquil. 